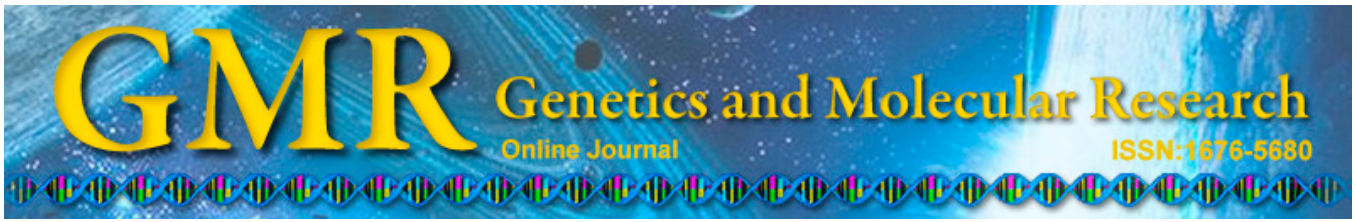

\title{
Relationship between serum adiponectin and osteoprotegerin levels and coronary heart disease severity
}

\author{
H.L. Zhang and X. Jin \\ Department of Cardiology, \\ Affiliated Hospital of Shandong Jining Medical College, \\ Jining, Shandong, China \\ Corresponding author: H.L. Zhang \\ E-mail: HuilingZhang123@126.com
}

Genet. Mol. Res. 14 (3): 11023-11029 (2015)

Received February 20, 2015

Accepted June 8, 2015

Published September 21, 2015

DOI http://dx.doi.org/10.4238/2015.September.21.15

\begin{abstract}
This study aimed to explore serum adiponectin and osteoprotegerin levels in patients with coronary heart disease (CHD) and their correlation with inflammatory and ischemia factors. From September 2010 to Augest 2010, 347 CHD patients were enrolled for a retrospective analysis. Serum lipoprotein phospholipase A2 (Lp-PLA2), hypersensitive C-reactive protein (hs-CRP), ischemia modified albumin (IMA), and adiponectin and osteoprotegerin levels were detected and analyzed. Serum adiponectin levels $(\mathrm{ng} / \mathrm{mL}, \mathrm{CV}$ was $4.3 \%$ at $250 \mathrm{ng} /$ $\mathrm{mL})$ were found to be negatively correlated with Lp-PLA2 $(\mathrm{r}=-0.958$, $\mathrm{P}=0.014)$ and hs-CRP $(\mathrm{r}=-0.958, \mathrm{P}=0.015)$ and positively correlated with IMA $(r=0.962, P=0.025)$. Serum osteoprotegerin levels were positively correlated with Lp-PLA2 $(\mathrm{r}=0.933, \mathrm{P}=0.027)$ and hs-CRP $(r=0.932, P=0.022)$ and negatively correlated with IMA $(r=-0.924$, $\mathrm{P}=0.017$ ). In addition, serum adiponectin levels negatively correlated with osteoprotegerin levels. In conclusion, serum adiponectin level was negatively correlated with CHD progression, whereas serum
\end{abstract}


osteoprotegerin level was positively correlated with CHD progression. Combined detection of adiponectin and osteoprotegerin levels may be of potential value in the clinical determination of CHD severity.

Key words: Adiponectin; Osteoprotegerin; Coronary heart disease; Inflammatory factor; Ischemia factor

\section{INTRODUCTION}

Coronary heart disease (CHD) is primarily caused by lipid metabolism disorder that leads to a large number of atheromatous unmetabolized lipid accumulates. These adsorb to the arterial intima if CHD worsens, hampering blood flow and causing heart ischemia and angina.

Adiponectin is an active polypeptide protein that acts in an autocrine manner and regulates lipid metabolism and anti-inflammatory activity. The occurrence of cardiovascular disease, especially CHD, is related to lipid metabolism disorders and inflammation. Therefore, adiponectin may be associated with CHD occurrence. Numerous studies have shown that adiponectin levels are correlated with hypertension, CHD, and diabetes, among other conditions (Iwashima et al., 2006; Wang and Scherer, 2008). Osteoprotegerin, a cytokine secreted by osteoclasts, can bind with the Receptor Activator of Nuclear factor-kappa B (RANK) expressed by osteoclasts to promote osteoclast differentiation, increase mature osteoclast activity, and inhibit osteoclast apoptosis (Simonet et al., 1997; Udagawa et al., 2000). Osteoprotegerin in the serum has a critical role in regulating the occurrence of cardiovascular diseases. Previous studies have suggested that osteoprotegerin function is associated with atherosclerosis and calcification (Schoppet et al., 2003; Shin et al., 2006). In the present study, we investigated serum adiponectin and osteoprotegerin levels and their correlation with inflammatory and ischemia factors in CHD, and revealed the relationship between adiponectin and osteoprotegerin.

\section{MATERIAL AND METHODS}

\section{Clinical samples}

A total of 347 CHD patients (187 men, 160 women; mean age, $64.0 \pm 4.3$ years) were enrolled from August 2010 to September 2010 for a retrospective analysis. The mean concentrations of serum lipoprotein phospholipase A2 (Lp-PLA2), hypersensitive C-reactive protein (hs-CRP), and ischemia modified albumin (IMA) were $452.43 \pm 217.14 \mathrm{ng} / \mathrm{mL}, 13.63$ $\pm 9.82 \mathrm{mg} / \mathrm{L}$, and $62.46 \pm 10.38 \mathrm{kU} / \mathrm{L}$, respectively. The exclusion criteria were as follows: acute or chronic infections; trauma, surgery, or cerebrovascular accident over the past 15 days; combined serious pulmonary, hepatic, or renal insufficiency; malignant tumor or autoimmune disease; and recent short-term use of anti-inflammatory drugs.

\section{Serum specimen collection and detection}

All blood samples were obtained in the early morning hours before breakfast and centrifuged at $1000 \mathrm{~g}$ for $15 \mathrm{~min}$ to eliminate the blood cells. The supernatant was used for detection using enzyme-linked immunosorbent assay (ELISA). Serum samples were stored at $-20^{\circ} \mathrm{C}$ for later use. 
Lp-PLA2, hs-CRP, IMA, and adiponectin and osteoprotegerin levels in the serum were detected by ELISA according to the instructions in the manual. Specifically, the diaDexus PLAC ${ }^{\circledR}$ Test ELISA Kit (Cat No. 90123, DiaDexus, USA) was used to detect serum Lp-PLA2 concentration [ng/mL, percent of CV (coefficient of variation) was $9.8 \%$ at $125 \mathrm{ng} /$ $\mathrm{mL}$; the hs-CRP ELISA Kit (Cat No. EK-072-62, Phoenix Pharmaceuticals, USA) was used to detect serum hs-CRP concentration ( $\mathrm{ng} / \mathrm{mL}, \mathrm{CV}$ was $4.3 \%$ at $10 \mathrm{ng} / \mathrm{mL}$ ); the Chemistry Analyzer (Roche Cobas Mira Plus, Switzerland) was used to detect serum IMA concentration (kU/L); the Adiponectin ELISA Kit (Cat No.10-1193-01, Mercodia, USA) was used to detect serum adiponectin concentration $(\mathrm{ng} / \mathrm{mL}, \mathrm{CV}$ was $4.3 \%$ at $250 \mathrm{ng} / \mathrm{mL})$; and the Osteoprotegerin ELISA Kit (Cat No. ELH-OPG-001, RayBiotech, USA) was used to detect serum osteoprotegerin concentration ( $\mathrm{ng} / \mathrm{mL}, \mathrm{CV}$ was $4.4 \%$ at $50 \mathrm{ng} / \mathrm{mL}$ ).

\section{Statistical analysis}

Statistical analysis was conducted using the $t$-test and Pearson correlation analysis with the Statistical Package for Social Sciences System Version 17.0 (SPSS, Chicago, IL, USA).

\section{RESULTS}

\section{Correlation between adiponectin and CHD-related inflammatory and ischemia factors}

Serum Lp-PLA2, hs-CRP, and IMA concentrations were considered to be the evaluation indexes for CHD severity. Following detection of serum adiponectin, Lp-PLA2, hs-CRP, and IMA levels in 347 patients, a correlation analysis was performed (Figure 1). The adiponectin level had a significant negative correlation with Lp-PLA2 $(r=-0.734, \mathrm{P}=0.014)$ and hs-CRP levels $(\mathrm{r}=-0.624, \mathrm{P}=0.015)$, and positively correlated with the IMA level $(\mathrm{r}=0.743, \mathrm{P}=0.025)$.
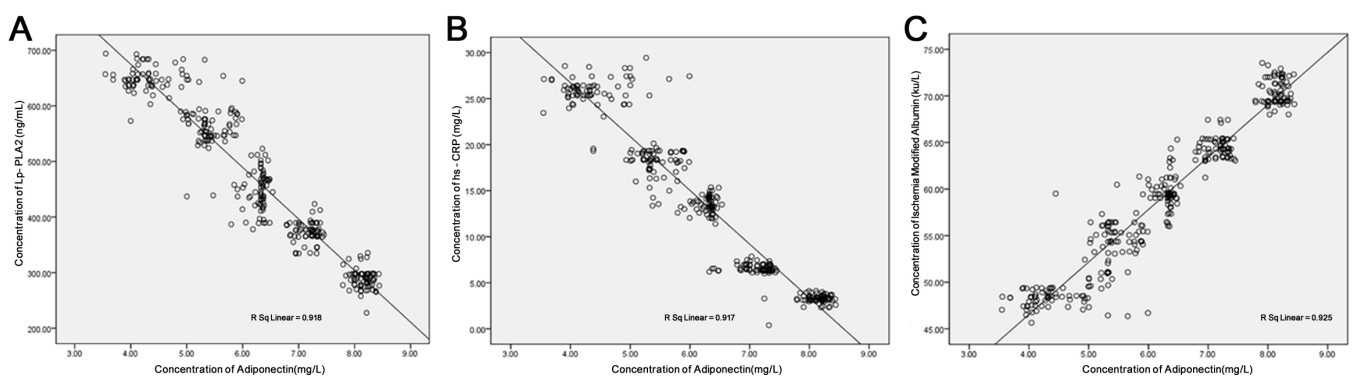

Figure 1. Correlation analysis between serum adiponectin level and LP-PLA2, hs-CRP, and IMA levels in CHD. A. Correlation analysis between adiponectin and LP-PLA2. B. Correlation analysis between adiponectin and hs-CRP. C. Correlation analysis between adiponectin and IMA.

\section{Correlation between osteoprotegerin and CHD-related inflammatory and ischemia factors}

Following detection of serum osteoprotegerin, Lp-PLA2, hs-CRP, and IMA levels in 
347 patients, a correlation analysis was performed (Figure 2). The osteoprotegerin level had remarkable positive correlation with Lp-PLA2 $(\mathrm{r}=0.614, \mathrm{P}=0.027)$ and hs-CRP levels $(\mathrm{r}=$ $0.621, \mathrm{P}=0.022)$ and a negative correlation with the IMA level $(\mathrm{r}=-0.723, \mathrm{P}=0.017)$.
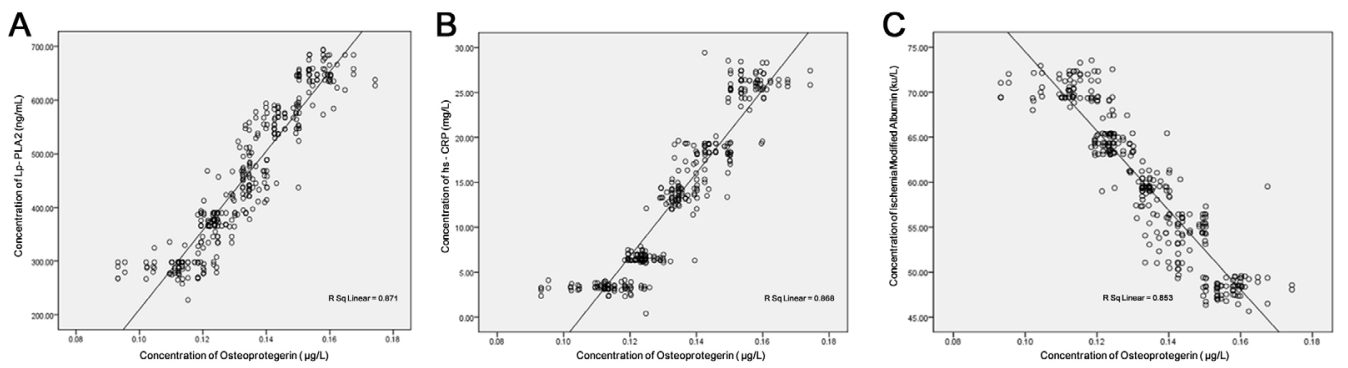

Figure 2. Correlation analysis between serum osteoprotegerin level and LP-PLA2, hs-CRP, and IMA levels in CHD. A. Correlation analysis between osteoprotegerin and LP-PLA2. B. Correlation analysis between osteoprotegerin and hs-CRP. C. Correlation analysis between osteoprotegerin and IMA.

\section{Correlation between adiponectin and osteoprotegerin}

To further investigate the relationship between adiponectin and osteoprotegerin levels in different types of CHD patients, a correlation analysis was performed (Figure 3). The osteoprotegerin expression level decreased following the upregulation of adiponectin, and a statistically significant negative correlation $(\mathrm{r}=-0.731, \mathrm{P}=0.036)$ was revealed between the two.

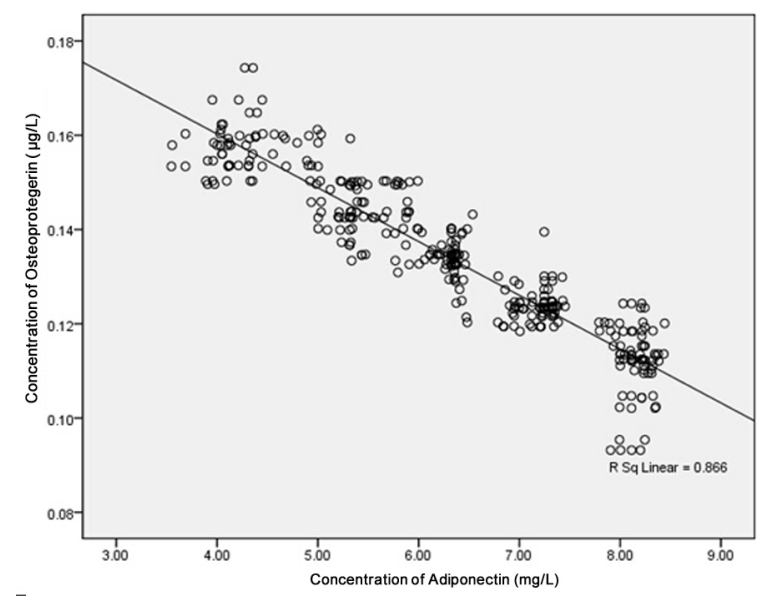

Figure 3. Correlation analysis between adiponectin and osteoprotegerin.

\section{DISCUSSION}

Several clinical studies have suggested that the severity of CHD is significantly and negatively correlated with Lp-PLA2, hs-CRP, and IMA protein levels in the peripheral blood (Dada et al., 2002; Erbagci et al., 2002; Shah et al., 2009; Charpentier et al., 2010). Many studies 
have also shown that inflammatory and ischemia factors are predictors of CHD severity. In the present study, serum inflammatory and ischemia factors were used to quantify the severity of CHD. Therefore, the relationship between adiponectin and osteoprotegerin levels and the severity of CHD can be indirectly evaluated using Lp-PLA2, h s-CRP, and IMA expression levels.

Adiponectin is a cytokine secreted by adipose tissue. It belongs to the collagen superfamily containing four structure domains: the amino terminal signal sequence, collagenoid structure domain, nonhomologous sequences, and spherical structure domain at the carboxyl terminal (Min et al., 2012). Yamauchi et al. (2003) cloned two key adiponectin receptors including AdipoR1 and AdipoR2, and found them overexpressed in the thrombus. This may indicate that the receptor-associated ligand adiponectin may participate in the hardening process of atherosclerosis, potentially associated with coronary artery disease. Adiponectin has been proven to be closely related to cardiovascular disease and lipoprotein metabolism. Both animal and human experiments showed that the plasma adiponectin level decreased in CHD patients (El-Mesallamy et al., 2012). Studies have shown that adiponectin has an apparent correlation with atherosclerosis (Hasan-Ali et al., 2011) and insulin resistance (Kishida et al., 2012). It is an independent risk factor for CHD occurrence and has a close relationship with the prognosis of CHD (Baldasseroni et al., 2012). Chan et al. (2005) investigated the correlation between adiponectin and multiple molecules such as cholesterol and triglycerides, and found that adiponectin can directly affect the metabolism of these molecules resulting in impact cardiovascular disease occurrence and progress by regulating lipid metabolism. Therefore, it is suggested that adiponectin could become an independent factor for CHD occurrence and development. Our study investigated the correlation between serum adiponectin level and CHD related factors in different types of CHD patients to obtain an indirect quantitative analysis of the correlation. Our results were consistent with that of the study by Frystyk et al. (2007).

Osteoprotegerin not only reduces osteoclast differentiation and increases bone mineral density, it is also an important vascular regulating factor closely related to atherosclerosis and calcification occurrence. Specially, it may play an important role in regulating CHD development. A previous study has found that osteoprotegerin levels were closely associated with CHD and had a potentially significant effect on prognosis (Celczynska Bajew et al., 2011). Walus-Miarka et al. (2011) indicated that osteoprotegerin can affect atherosis progression in patients with diabetes combined with atherosis. Avignon et al. (2007) found that osteoprotegerin expression level increased significantly in the serum of patients with diabetes combined with CHD. Our results showed that the osteoprotegerin expression level had a significant positive correlation with CHD severity, which was in agreement with the above reports. However, there is controversy regarding osteoprotegerin being considered an independent factor for $\mathrm{CHD}$ occurrence and development. Atherosclerosis and vascular calcification was found significantly accelerated in osteoprotegerin knockout mice, which suggested that osteoprotegerin may inhibit atherosclerotic lesions and calcification formation (Bennett et al., 2006). This, however, needs to be investigated further.

Finally, the correlation between adiponectin and osteoprotegerin in CHD was also discussed in the present study. Serum adiponectin and osteoprotegerin levels were found to be negatively correlated in the analysis of the 347 CHD patients. Previous studies have reported that adiponectin may inhibit osteoprotegerin expression in human tissue via the AdR1/p38 MARK signaling pathway (Luo et al., 2006), and this explains our results. Several studies 
have concluded that adiponectin is the signal of CHD, which indicates that adiponectin can be treated as an independent diagnostic factor (Ai et al., 2011). Our results showed that detection of both adiponectin and osteoprotegerin may serve as a potential evaluation of CHD severity.

\section{Conflicts of interest}

The authors declare no conflict of interest.

\section{ACKNOWLEDGMENTS}

We thank the anonymous reviewers for reviewing this manuscript.

\section{REFERENCES}

Ai M, Otokozawa S, Asztalos BF, White CC, et al. (2011). Adiponectin: an independent risk factor for coronary heart disease in men in the Framingham offspring Study. Atherosclerosis 217: 543-548.

Avignon A, Sultan A, Piot C, Mariano-Goulart D, et al. (2007). Osteoprotegerin: a novel independent marker for silent myocardial ischemia in asymptomatic diabetic patients. Diabetes Care 30: 2934-2939.

Baldasseroni S, Mannucci E, Orso F, Di Serio C, et al. (2012). Adiponectin in outpatients with coronary artery disease: independent predictors and relationship with heart failure. Nutr. Metab. Cardiovasc. Dis. 22: 292-299.

Bennett BJ, Scatena M, Kirk EA, Rattazzi M, et al. (2006). Osteoprotegerin inactivation accelerates advanced atherosclerotic lesion progression and calcification in older ApoE-/- mice. Arterioscler. Thromb. Vasc. Biol. 26: 2117-2124.

Celczynska Bajew L, Horst Sikorska W, Bychowiec B, Wykretowicz A, et al. (2011). The effects of osteoprotegerin (OPG) gene polymorphism in patients with ischaemic heart disease on the morphology of coronary arteries and bone mineral density. Kardiol. Pol. 69: 573-578.

Chan DC, Watts GF, Ng TW, Uchida Y, et al. (2005). Adiponectin and other adipocytokines as predictors of markers of triglyceride-rich lipoprotein metabolism. Clin. Chem. 51: 578-585.

Charpentier S, Ducasse JL, Cournot M, Maupas-Schwalm F, et al. (2010). Clinical assessment of ischemia-modified albumin and heart fatty acid-binding protein in the early diagnosis of non-ST-elevation acute coronary syndrome in the emergency department. Acad. Emerg. Med. 17: 27-35.

Dada N, Kim NW and Wolfert RL (2002). Lp-PLA2: an emerging biomarker of coronary heart disease. Expert Rev. Mol. Diagn. 2: 17-22.

El-Mesallamy HO, Hamdy NM, Salman TM and Ibrahim SM (2012). Adiponectin and sE-selectin concentrations in relation to inflammation in obese type 2 diabetic patients with coronary heart disease. Angiology 63: 96-102.

Erbagci AB, Tarakcioglu M, Aksoy M, Kocabas R, et al. (2002). Diagnostic value of CRP and Lp(a) in coronary heart disease. Acta Cardiol. 57: 197-204.

Frystyk J, Berne C, Berglund L, Jensevik K, et al. (2007). Serum adiponectin is a predictor of coronary heart disease: a population-based 10-year follow-up study in elderly men. J. Clin. Endocrinol. Metab. 92: 571-576.

Hasan-Ali H, Abd El-Mottaleb NA, Hamed HB, Abd-Elsayed A, et al. (2011). Serum adiponectin and leptin as predictors of the presence and degree of coronary atherosclerosis. Coron. Artery Dis. 22: 264-269.

Iwashima Y, Horio T, Kumada M, Suzuki Y, et al. (2006). Adiponectin and renal function, and implication as a risk of cardiovascular disease. Am. J. Cardiol. 98: 1603-1608.

Kishida K, Funahashi T and Shimomura I (2012). Molecular mechanisms of diabetes and atherosclerosis: role of adiponectin. Endocr. Metab. Immune Disord. Drug Targets 12: 118-131.

Luo XH, Guo LJ, Xie H, Yuan LQ, et al. (2006). Adiponectin stimulates RANKL and inhibits OPG expression in human osteoblasts through the MAPK signaling pathway. J. Bone Miner. Res. 21: 1648-1656.

Min X, Lemon B, Tang J, Liu Q, et al. (2012). Crystal structure of a single-chain trimer of human adiponectin globular domain. FEBS Lett. 586: 912-917.

Schoppet M, Sattler AM, Schaefer JR, Herzum M, et al. (2003). Increased osteoprotegerin serum levels in men with coronary artery disease. J. Clin. Endocrinol. Metab. 88: 1024-1028. 
Shah T, Casas JP, Cooper JA, Tzoulaki I, et al. (2009). Critical appraisal of CRP measurement for the prediction of coronary heart disease events: new data and systematic review of 31 prospective cohorts. Int. J. Epidemiol. 38: 217 231.

Shin JY, Shin YG and Chung CH (2006). Elevated serum osteoprotegerin levels are associated with vascular endothelial dysfunction in type 2 diabetes. Diabetes Care 29: 1664-1666.

Simonet WS, Lacey DL, Dunstan CR, Kelley M, et al. (1997). Osteoprotegerin: a novel secreted protein involved in the regulation of bone density. Cell 89: 309-319.

Udagawa N, Takahashi N, Yasuda H, Mizuno A, et al. (2000). Osteoprotegerin produced by osteoblasts is an important regulator in osteoclast development and function. Endocrinol. 141: 3478-3484.

Walus-Miarka M, Katra B, Fedak D, Czarnecka D, et al. (2011). Osteoprotegerin is associated with markers of atherosclerosis and body fat mass in type 2 diabetes patients. Int. J. Cardiol. 147: 335-336.

Wang ZV and Scherer PE (2008). Adiponectin, cardiovascular function, and hypertension. Hypertension 51: 8-14.

Yamauchi T, Kamon J, Ito Y, Tsuchida A, et al. (2003). Cloning of adiponectin receptors that mediate antidiabetic metabolic effects. Nature 423: 762-769. 